\title{
Indistinguishable elements in the origins of quantum statistics. The case of Fermi-Dirac statistics
}

\author{
Enric Pérez ${ }^{\mathrm{a}}(\mathbb{D})$ and Joana Ibáñez ${ }^{\mathrm{b}}$ \\ Departament de Física de la Matèria Condensada, Universitat de Barcelona, Carrer de Martí i Franquès 1, \\ 08028 Barcelona, Spain
}

Received 20 July 2021 / Accepted 22 November 2021 / Published online 5 January 2022

(C) The Author(s) 2021

\begin{abstract}
In this paper, we deal with the historical origins of Fermi-Dirac statistics, focusing on the contribution by Enrico Fermi of 1926. We argue that this statistics, as opposed to that of Bose-Einstein, has been somewhat overlooked in the usual accounts of the old quantum theory. Our main objective is to offer a critical analysis of Fermi's seminal paper and its immediate impact. Secondly, we are also interested in assessing the status of the particle concept in the years 1926-1927, especially regarding the germ of quantum indistinguishability. We will see, for example, that the first applications of the Fermi-Dirac statistics to the study of metals or stellar matter had a technical nature, and that their main instigators barely touched upon interpretative matters. Finally, we will discuss the reflections and remarks made in these respects in two famous events in physics of 1927, the Como conference and the fifth Solvay congress.
\end{abstract}

\section{Introduction}

There are many interesting historiographical studies on the origin of Bose-Einstein statistics and the birth of the concept of indistinguishable particle $[2,36]$, an issue being still debated today $[8,44]$. In contrast, the origins of the Fermi-Dirac statistics have been only marginally addressed; accounting for that asymmetry in the historiographical literature on quantum theory is one of the questions that we aim to address. To that end, in this paper, we propose to analyze in detail the historical origins of this statistics, focusing on the contribution of the renowned Italian physicist Enrico Fermi [23]. In historiographical publications devoted especially to Fermi's contribution, physical questions are somewhat neglected or not discussed in any great depth $[3,4,7,45,46]$. We will attempt to go into technical details and to correct this omission in order to complete the narrative of the old quantum theory, especially regarding the birth of quantum statistics. Our main task is to offer a critical analysis of Fermi's contribution, and for this purpose, reading it in the light of the first steps of the other statistics - those of Bose-Einstein - has proved very useful.

This comparison will help us to appreciate the extent to which Fermi was not inspired by Einstein's work, as we will argue that he did not make use of the detailed remarks by the father of relativity on the new statistics, resulting from his reflections over a period of more than twenty years. In other words, Fermi did not make use of the enlightening effect that Bose's combinatorics had on Einstein. Hence, we will use this essay to return once more to the thorny issue of indistinguishable particles with the help of history. How did the birth of fermion statistics contribute to the emergence of quantum indistinguishability?

We are also interested in presenting the state of the particle concept in those first steps of the new statistics. It is clear that the concept of 'indistinguishable particle' took years to crystallize, and we will argue that by late 1926 the concept of particle had hardly suffered any damage. That is, in general terms it was still being used as a visualizable and non-problematic notion. Moreover, just as there was an attempt - in Einstein's case - to find an explanation for the lack of statistical independence (in the form of the de Broglie hypothesis, for example [40]), Fermi did not seem to care at all. To broaden our study, we will also consider the first applications of Fermi statistics, to investigate the extent to which it was seen as a revolutionary or puzzling contribution, especially with regard to the individuality of particles. We will see, for example, that its applications to the study of metals had a practical, technical nature, and that the main instigators of this study, Wolfgang Pauli and Arnold Sommerfeld, barely touched upon interpretative matters, at least in this context. We will also comment on Ralph H. Fowler's contributions, in which the English physicist scarcely emphasized the conceptual implications of the new statistics.

\footnotetext{
a e-mail: enperez@ub.edu (corresponding author)

b e-mail: joana_ibanez@hotmail.com
} 
It is worth noting that they were all physicists who belonged to a community that was at the time involved in the program of developing the new mechanics (Pauli's and Sommerfeld's close links with Heisenberg, for example, as well as those between Fowler and Dirac, are widely known). In fact, Fowler produced part of his work while in Copenhagen, and he expressed his gratitude for conversations he had with Niels Bohr on this matter. This forces us to consider that these were not isolated developments, as in Fermi's case, but the result of debates and meetings between physicists who, at that moment, were constructing quantum mechanics.

We will not go into too many biographical or contextual details, as these have been widely covered elsewhere $[46,50]$. For our purposes, a schematic background description of the academic situation of Fermi, the protagonist of our essay, is sufficient. He obtained his doctorate in Pisa in 1922. Shortly before that he had already begun to publish in national and international journals on various topics in physics, including mechanics, statistics, relativity, quantum theory, theory of solids, electromagnetism and other subjects. By the time he published his paper on the application of Pauli principle to the theory of ideal gases, in May 1926, Fermi had published some thirty essays. Previously, in 1923, he had gone to Göttingen for eight months, thanks to a scholarship from the Italian Ministry of Education, to work with the Born group. When he returned to Italy he taught mathematics in Rome for an academic year, and in September 1924 he traveled to Leiden (this time on a grant from the International Educational Board) to spend four months with Ehrenfest and his collaborators, which during that period included Einstein. After his return to Italy, Fermi obtained a temporary teaching position in Florence, until the end of 1926. Then in 1927, he obtained a post as a professor at La Sapienza, Rome. It was during his stay in Rome that he headed an international research group focusing primarily on nuclear physics. The paper that concerns us here was written while he was in Florence.

We will start by analyzing and commenting on Fermi's article (Sect. 2), which is the main object of our study, to subsequently go on to look for precedents and motivations in Fermi's previous research (Sect. 3); how did he come, for example, to consider the relevance of Pauli principle to the quantum theory of ideal gases? We will continue by comparing his approach with those of some of his contemporaries, especially Einstein (Sect. 4), and though we will leave for later publications a detailed discussion of the fundamental contributions made by Paul Dirac and Heisenberg, we will mention them [9,29]. Finally (Sect. 5), before summarizing our final remarks, we will focus on a discussion of how Fermi's statistics was received and how it was understood among the first people who developed and applied it.

\section{On the quantization of a monatomic ideal gas}

The article in which Fermi introduces the new statistics is a short piece, not divided into sections, and published in Zeitschrift für Physik in May 1926 [23]. It had been preceded by a shorter one that Fermi sent to the Academia dei Lincei in Rome in February [22]. The abstract makes the motivation clear: if Nernst's theorem is to remain valid for ideal gases at low temperatures, then the classical ideal gas law cannot be correct in that range. And the reason for the degeneration of the gas must be found in the quantization of molecular motions. Furthermore - the abstract continues - any degenerate gas theory involves some arbitrary assumptions about the statistical behavior of the molecules or their quantization. In the present work $[23, \text { p. 186] }]^{1}$ :

... only the assumption made for the first time by Pauli and based on numerous spectroscopic facts, that in a system there can never be two equivalent elements whose quantum numbers completely coincide, is used.

Under this hypothesis, Fermi would derive a new equation of state, as well as expressions for the internal energy and entropy of the gas, which for high temperatures coincide with the classical value. Note that in the abstract, the identity of the particles does not play any role.

In the introduction, Fermi already refers once again to Nernst's theorem, in particular to the tendency of specific heats to vanish at low temperatures. The following paragraph is worthy of note $[23$, p. 186]:

The purpose of the present work is to present a method for the quantization of the ideal gas, which in our opinion is as independent as possible from arbitrary assumptions about the statistical behavior of the gas molecules.

As we can see, Fermi insists on dissociating his contribution, as far as possible, from statistical hypotheses. He notes that recent attempts have been made to establish a new equation of ideal gases, and in a footnote he cites the works by Einstein and Planck [15,16,42]. Regarding the former, he states [23, p. 187, footnote 1]:

Our method is akin to Einstein's in that the assumption of the statistical independence of the molecules is abandoned in both methods, though the mode of dependence is completely different for us than in Einstein, and the final result for the deviations from the classical equation of state is even found to be in the opposite direction.

1 Unless stated otherwise, all translations are our own. 
Thus, Fermi explicitly establishes the lack of statistical independence in his approach, while differentiating it from the one postulated by Einstein. The formulas obtained by Planck, Einstein and Fermi are different, including the very equation of the ideal gases, but in his opinion it is not possible to experimentally distinguish between them.

After these brief, rather cursory comments on statistical matters, Fermi addresses the quantization issue. He states that various methods can be followed. For example, we can consider molecules colliding between parallel walls, or a gas subjected to an external field that forces atomic motions to be conditionally periodic. The ideality of the gas enables us to omit the interaction between molecules. However, this is not enough: the quantization obtained will become denser as we increase the dimensions of the container, until it becomes virtually continuous, and in a macroscopic container this would be the case. On this point, Fermi refers to an idea that he developed in a previous work [23, p. 187]:

By a quantitative calculation of this state of affairs, one can convince oneself that one only achieves a degeneration of the expected magnitude if one chooses a container so small that it contains on average only one molecule.

This idea came out in a paper of 1924 that we will discuss in more detail in the next section [21]. In that paper, in order to obtain the correct constant of entropy, Fermi imposes homogeneous spatial distribution on a gas by placing each particle in a volume $V / N$. Surely, in 1926, he is attempting to connect this earlier research with the current one and, in particular, with the central role played by the exclusion principle. It is interesting to note that the reference to that paper in the Italian version is accompanied by a quite different comment [22, p. 182]:

It therefore seems necessary to admit that some complement to Sommerfeld rules is needed for the calculation of systems which, like ours, contain elements that cannot be distinguished from each other.

The German version is confusing [23, p. 187], if not wrong, because it suggests that in the paper of 1924, we will find the missing rule required to obtain the correct degeneration, and that is not the case. In fact, in that paper, Fermi obtained the Sackur-Tetrode equation.

What is clear is that some other condition must be added to Sommerfeld's quantization rules when dealing with elements that are indistinguishable from each other, which is indeed what Fermi argued in 1924. In the Italian version, the inspiration for this additional condition comes from systems that, like the ideal gas, contain "identical elements": atoms with more than one electron. This is how he introduces the exclusion principle. The appropriateness of the analogy is based on the fact that the nuclear attraction of atomic electrons is much greater than the interaction between them (in the German version we do not find this justification necessary to neglect the Coulombian interaction). Hence, Fermi states that the additional condition to be added to Sommerfeld's rule shall be the Pauli principle: in an atom there cannot be two electrons with the same values for their quantum numbers. He will show that this rule, so useful in the field of spectroscopy, is also applicable in the treatment of the degenerate ideal gas: there will not be two molecules with the same quantum numbers. Needless to say, the indistinguishability of elements has, in this context, the same meaning as in classical statistics.

Fermi continues his presentation by taking a somewhat surprising step: he considers an external field (an elastic central field with spherical symmetry) that allows him to treat the motions of the molecules as conditionally periodic. Accordingly, the molecules carry out harmonic motions and the density of the gas will decrease as we move away from the center of attraction. The restoring force is:

$$
4 \pi^{2} \nu^{2} m r
$$

( $\nu$ is the frequency of the restoring force, $m$ the mass of the molecules and $r$ the distance to the center of attraction). Fermi dispenses with the internal degrees of freedom, which he assumes either do not exist or are in the ground state. So we have monatomic molecules, and the energy will be:

$$
\omega=h \nu\left(s_{1}+s_{2}+s_{3}\right)
$$

where $s_{1}, s_{2}, s_{3}$ are the usual quantum numbers of the harmonic oscillator. According to Pauli principle, two atoms will not share the same triple of numbers, and every value:

$$
s=s_{1}+s_{2}+s_{3}
$$

can be configured in:

$$
Q_{s}=\frac{(s+1)(s+2)}{2}
$$


ways. $^{2}$ Therefore, at most, we will have $Q_{s}$ molecules with total number $s$. Consequently, for zero temperature the total energy would not be zero. Fermi comments on this via an analogy with the atomic shell structure, highlighting once again spectroscopic investigations as a source of inspiration.

Next, Fermi focuses on tackling how to configure a general state of energy $E h \nu$, where $E$ is an integer and $N_{s}$ the number of molecules with energy $\operatorname{sh} \nu\left(N_{s} \leq Q_{s}\right)$. It is necessary to impose the constrains related to the total energy and the number of molecules:

$$
\begin{aligned}
\sum N_{s} & =N \\
\sum s N_{s} & =E .
\end{aligned}
$$

Fermi continues:

Two arrangements should be regarded as the same if the positions occupied by the molecules are the same: two arrangements which differ only in the permutation of the molecules in their positions are therefore to be regarded as the same arrangement. If two such arrangements were to be regarded as different, then one would multiply $P$ with the constant $N$ !: but one could easily see that this would have no influence on what follows.

This comment is puzzling as well as significant, as it shows clearly that Fermi was not taking Einstein's work as a standard for presenting his new treatment. If the aforementioned factor $N$ ! is introduced, following Einstein's method, the entropy does vary because taking a constant makes it impossible to affirm that Nernst's theorem is fulfilled. We will return to this point in Sect. 4.2. But Fermi took another route.

When we consider the case for $Q_{s}$ places and $N_{s}$ molecules, the possible configurations are:

$$
\left(\begin{array}{c}
Q_{s} \\
N_{s}
\end{array}\right)=\frac{Q_{s} !}{N_{s} !\left(Q_{s}-N_{s}\right) !}
$$

If we now take into consideration all the energy levels $i$, the number of configurations $[$ Anordnungen] $P$ we have is:

$$
P=\left(\begin{array}{l}
Q_{o} \\
N_{\circ}
\end{array}\right)\left(\begin{array}{l}
Q_{1} \\
N_{1}
\end{array}\right)\left(\begin{array}{l}
Q_{2} \\
N_{2}
\end{array}\right) \cdots=\prod_{s}\left(\begin{array}{l}
Q_{s} \\
N_{s}
\end{array}\right)
$$

To obtain the maximum of $P$ paying attention to constrains (1) and (2), Fermi proceeds as usual: he applies Stirling approximation and uses the method of Lagrange multipliers ( $\alpha$ and $\beta$ in the following expression). He arrives at:

$$
N_{s}=Q_{s} \frac{\alpha e^{-\beta s}}{1+\alpha e^{-\beta s}}
$$

and hence:

$$
\begin{aligned}
N & =\sum_{0}^{\infty} Q_{s} \frac{\alpha e^{-\beta s}}{1+\alpha e^{-\beta s}} \\
\frac{W}{h \nu} & =E=\sum_{0}^{\infty} s Q_{s} \frac{\alpha e^{-\beta s}}{1+\alpha e^{-\beta s}}
\end{aligned}
$$

where $W$ is the total energy, and $\alpha$ and $\beta$ Lagrange multipliers.

\subsection{Introduction of temperature and equation of state}

In any statistical treatment, the introduction of temperature is a crucial and delicate point as well, and in this case it illustrates Fermi's approach to the problem quite well. Moreover, note that he is dealing with a non-homogeneous system (a gas under an elastic central field), which makes the definition of intensive variables problematic. Fermi argues that there are two possible paths. One through Boltzmann's principle:

$$
S=\kappa \log P
$$

${ }^{2}$ In usual combinatorics terms, we are dealing here with combinations with repetition of 3 elements taken $s$ by $s, C R_{3, s}$. 
and the thermodynamic relation

$$
T=\frac{d W}{d S} .
$$

This would be, with a few nuances that we will discuss later, Einstein's method. In Fermi's opinion, it has a disadvantage: it "needs a more or less arbitrary approach for the probability of a state" [23, p. 191]. To avoid this procedure, Fermi will take the other path, which again highlights the extent to which he did not intend to introduce physical statistical innovations. As the density of the gas will depend on the distance to the center, at very large distances the effects of degeneracy will vanish and we will find Maxwell-Boltzmann distribution (which also implies spatial homogeneity). Thus, we can determine the temperature in that regime and, since by definition the gas is in thermodynamic equilibrium, we will know it for all the gas.

Accordingly, Fermi proceeds to determine the density of particles (per unit volume) with a certain kinetic energy between $L$ and $d L$. He obtains, of course, a dependency with the distance to the equilibrium point $r$ :

$$
n(L) d L=n_{s} d s=\frac{2 \pi(2 m)^{3 / 2}}{h^{3}} \sqrt{L} d L \frac{\alpha e^{-\frac{2 \pi^{2} \nu m \beta r^{2}}{h}} e^{-\frac{\beta L}{h \nu}}}{1+\alpha e^{-\frac{2 \pi^{2} \nu m \beta r^{2}}{h}}} e^{-\frac{\beta L}{h \nu}},
$$

which he compares with the known (classical) formula:

$$
n^{*}(L) d L=K \sqrt{L} d L e^{-L / \kappa T}
$$

( $\kappa$ is Boltzmann's constant and $K$ a proportionality constant). For both expressions to match for very large $r$ 's, we just need to identify:

$$
\beta=\frac{h \nu}{\kappa T} .
$$

Finally, (6) can be written as:

$$
n(L) d L=\frac{(2 \pi)(2 m)^{3 / 2}}{h^{3}} \sqrt{L} d L \frac{A e^{-L / \kappa T}}{1+A e^{-L / \kappa T}},
$$

where

$$
A=\alpha e^{-\frac{2 \pi^{2} \nu^{2} m r^{2}}{\kappa T}} .
$$

The total density of particles is:

$$
n=\int_{0}^{\infty} n(L) d L=\frac{(2 \pi m \kappa T)^{3 / 2}}{h^{3}} F(A),
$$

with

$$
F(A)=\frac{2}{\sqrt{\pi}} \int_{0}^{\infty} \frac{A \sqrt{x} e^{-x} d x}{1+A e^{-x}},
$$

and the mean kinetic energy:

$$
\bar{L}=\frac{1}{n} \int_{0}^{\infty} \operatorname{Ln}(L) d L=\frac{3}{2} \kappa T \frac{G(A)}{F(A)},
$$

with:

$$
G(A)=\frac{4}{3 \sqrt{\pi}} \int_{0}^{\infty} \frac{A x^{3 / 2} e^{-x} d x}{1+A e^{-x}} .
$$

As usual, in (8) we can determine $A$ in terms of density and temperature. By substituting that in (7) and (9), we can obtain the distribution of velocities and kinetic energies in terms of density and temperature. All this through power series. 
In order to calculate the pressure, Fermi resorts to the Virial theorem:

$$
p=\frac{2}{3} n \bar{L}=\kappa T \frac{G(A)}{F(A)} .
$$

Again, to obtain a result we have to expand functions $F(A)$ and $G(A)$ in power series for $A$ greater and lower than unity. For a weakly degenerated gas $(A<<1)$ :

$$
p=n \kappa T\left\{1+\frac{1}{10} \frac{h^{3} n}{(\pi m \kappa T)^{3 / 2}}+\cdots\right\}
$$

that is, the pressure of a quantum ideal gas that fulfills the exclusion principle is greater than that of a classical ideal gas. For a gas at $5^{\circ}$ Kelvin with a pressure of 10 atmospheres and with the atomic weight of helium, the increase is $15 \%{ }^{3}$ Finally, for a gas with $A>>1$, Fermi obtains the expansions for pressure and mean kinetic energy and verifies that the ground state is degenerate with a zero point energy. He also calculates the heat capacity and checks that it satisfies Nernst's principle, in addition to showing that it is proportional to temperature at very low temperatures.

Fermi closes the paper by comparing his results with the entropy of an ideal gas deduced by Stern and Tetrode $[56,59]$. He does not use Boltzmann's principle, but calculates the entropy through:

$$
S=n \int_{0}^{T} \frac{d \bar{L}}{T}
$$

leading up to:

$$
S=n \kappa\left\{\frac{5}{2} \frac{G(A)}{F(A)}-\log A\right\} .
$$

Once again, the constant $A$ must be obtained from (8). Having done this in the classical limit, Fermi obtains the well-known formula for the entropy of an ideal classical gas:

$$
S=n \kappa\left\{\log \frac{(2 \pi m \kappa T)^{3 / 2}}{n h^{3}}+\frac{5}{2}\right\} .
$$

\subsection{Additional remarks}

Let us highlight a few points that deserve further comment and attention before we move on to the antecedents of the paper. To begin with, note that Fermi connects the ideal gas and multielectronic atoms through spectroscopy and, more specifically, through the Pauli principle. To do this, he ignores the Coulombian interaction between electrons, as he explicitly stated in the Italian version and did not in the German one. Despite the fact that the interaction with the nucleus is much greater (greater the heavier the atom and the inner the electrons under consideration; [22], pp. 182-183), it does not seem enough to justify an analogy between neutral free molecules and bound electrons. Hence, it must be conceived simply as an heuristic inspiration.

Fermi's use of a harmonic potential is also remarkable. Certainly, this procedure does not influence the final result and allows him to consider the system properly quantized, according to the quantization rules of the old quantum theory. The expression to be maximized (4) corresponds to a system of oscillators, not to free particles. It is therefore logical that there has been a great deal of speculation regarding this point among the few historians of physics that have addressed this paper. We disagree with De Gregorio and Sebastiani's suggestion about the importance of using the harmonic potential to make use of the adiabatic hypothesis $[7$, p. 8], mostly because it has no role in this work. For G. Parisi, the reason for dealing with harmonic motions is to be able to perform the classical limit in a simpler way [4, p. 72, footnote 8]: far from the equilibrium point the density is low and the classical results must be recovered. However, Einstein's earlier work easily showed how to perform the classical limit without introducing a potential that would break homogeneity and introduce dubious assumptions.

Indeed, the role of the external field introduced by Fermi is to deal with known quantized energies, as he explicitly admits. This shows again how far away Fermi's approach was from that of Einstein, since Einstein merely considered the quantization of the phase space, in accordance with Satyendra Nath Bose's proposal. As we

3 We obtain a $25 \%$ increase. 
will discuss below, paradoxical as it may seem, quantization of energy did not play any role in Einstein's theory. Let us note, for the rest, an additional peculiarity resulting from Fermi's approach: the volume does not explicitly appear in the expressions. The gas - as it occurs with the harmonic oscillator - is not confined. Apparently, this could alleviate the problem of the extensivity of the obtained quantities related to the famous term $N$ ! or some term of the type $N^{N} e^{a N}$ ( $a$ is a numerical factor), so that corrects the effect of a factor $V^{N}$. Notwithstanding, as was already the case with Bose-Einstein statistics, what really avoids the problem that leads to the Gibbs paradox in Fermi's treatment is the change of focus of the combinatorial problem from particles to monoparticular states. Or, in terms of ensembles, switching from canonical or microcanonical ensemble to the grandcanonical, where $N$ is no longer a variable (it has ceded its place to $\mu$, the chemical potential).

Finally, let us consider the issue of the identity of the particles. Fermi's statements in this regard are somewhat disconcerting. To begin with, in the abstract, he relates statistical and quantum hypotheses through the exclusion principle. But more significant is Fermi's comment in relation to considering two configurations differing only in permutations between particles. He states that those configurations should not be considered as different, but adds that were this to be done, it would not modify the final result. We will see below that had he followed Einstein's method - which is much more rigorous from a statistical point of view and, without a doubt, makes the new statistical properties more obvious - the result would be modified.

Fermi's application of the Virial theorem also exemplifies his attitude in this regard. Fermi uses it to obtain the equation of state, which shows that he still maintains the corpuscular interpretation of the pressure. Remember that this theorem was deduced by Clausius when trying to establish a connection between an adiabatic compression in a thermodynamic system and the energies of particles subjected to a certain potential. The theorem prescribes that:

$$
\bar{T}=-\frac{1}{2} \overline{\sum_{i} \overrightarrow{F_{i}} \cdot \overrightarrow{r_{i}}}
$$

where the horizontal lines indicate time averages, $T$ the kinetic energy, $\vec{F}_{i}$ the forces acting on the particles and $\overrightarrow{r_{i}}$ their positions. Its usefulness for finding equations of state depends entirely on the shape of the external potentials and the interaction between molecules. In the case of the ideal gas, the only existing force is that exerted by the container on the molecules. Fermi uses this result for his gas, despite the fact that it is not confined, and he does not comment on the possible influence of the elastic field on the Virial of the system. In the case of a three-dimensional harmonic potential such as that proposed by Fermi, these averages in each of the axes cancel out, so that the Virial is limited to the pressure of the walls on the gas. In short, Fermi relies on a (classical) mechanistic vision to obtain the equation of state for gas. We will see that Einstein avoided this procedure.

\section{Fermi's route}

We are going now to assess to what extent it makes sense to relate Fermi's previous contributions to the problem of the entropy constant of monatomic gases with the historical paper of 1926, his "first major contribution to physics" [50, p. 42]. We will base our analysis on the published work, because we have not found any other sources that allow us to trace Fermi's route. According to his friend and colleague Franco Rasetti (author of the comments that precede Fermi's paper in the volume of his complete works), Fermi, despite becoming the leader of the incipient group in Rome, was not very keen on explaining what he was up to [25, p. 178]. Still, Rasetti recalls having discussed the ideal gas with Fermi in 1924 and 1925, and that he aimed to find a new theory that gives an expression compatible with Nernst's principle and with the Sackur and Tetrode equation, all of which would be "free of the various arbitrary assumptions that it had been necessary to introduce in statistical mechanics in order to derive a correct entropy value." He also remembered that Fermi was not influenced by the work of Bose and Einstein. We will argue in what follows that these claims are more than plausible.

Fermi had turned his attention to the system of the ideal gas some years earlier, and in particular to the annoying problem of the $N$ factorial and the extensivity of entropy. In this respect, we completely agree with Belloni-and consequently disagree with Segrè - that the motivation behind the paper that we have just commented on was the statistics of various particles, multi-electronic atoms, and in no case the theory of metals [1]. It is also apparent that Fermi's stay in Göttingen in 1923 strongly influenced him. There, Born and some of his collaborators were studying topics very closely related to the problem of gases [46].

The question we will ask ourselves can be formulated more or less as follows. Can some kind of investigation or reflection by the Italian physicist be interpreted as his early intuition of the exclusion principle [39, 58]. According to Rasetti, there is no doubt that this is the case [25, p. 124]. In his opinion, Fermi had detected "something missing" when he proposed, as we shall see, enclosing the particles of an ideal gas in imaginary boxes of volume $V / N$; it is disputable, however, that this method has any relation to "the exchange of the coordinates of identical 
particles in the wave function of a system" [25, p. 124]. Rasetti even states that Fermi told Segrè that he would have come to the Pauli principle by himself [25, p. 178]. As Sebastiani and Cordella note [46, p. 91]:

[Fermi] told much later to Segrè that the division of phase space into finite cells had occupied him very much and that had not Pauli discovered the exclusion principle he might have arrived at it in a roundabout way from the entropy constant.

Hence, this connection between the spatial exclusion of molecules to obtain an acceptable entropy constant and Pauli principle starts, apparently, from Fermi himself. We will argue from the outset that one matter has little or nothing to do with the other. However, we will see that his interest in the problems that had accompanied the treatment of systems with identical particles for years, put him in a favorable situation to see in the exclusion principle a key element to elucidate them.

\subsection{Fermi on identical elements}

In December 1923 and January 1924, Fermi published two papers that make clear his familiarity with the problems surrounding the entropy constant of gases. These articles allow us to see the state of Fermi's ideas after having been at Göttingen (between January and August 1923) and before going to Leiden (last trimester of 1924). Both are short, and do not contain any general statements but instead, some very precise technical comments on the issues covered. The first deals with Stern's method for determining the absolute constant of the monatomic ideal gas, though Fermi does not determine it. He does in the second, but by following a completely different procedure.

In the paper entitled "On Stern's theory of the absolute constant of entropy of a perfect monatomic gas" [20] Fermi refers to the existing deductions of the Sackur and Tetrode formula for the entropy of an ideal gas:

$$
S=N \kappa\left\{\frac{5}{2} \ln T-\ln p+\ln \frac{(2 \pi m)^{3 / 2} \kappa^{5 / 2} e^{5 / 2}}{h^{3}}\right\}
$$

where $p$ is pressure. He highlights what for him is the dark spot that produces dissatisfaction among physicists with the available derivations: the introduction of quantization through the use of cells of volume $h^{3}$. Stern's method $[56,57]$ is preferable because $[20$, p. 115$]$ :

... [it] has the advantage of not making any of those unlawful hypotheses on the perfect gas, which are necessary

for the other authors, such as that of a quantization of the gas itself, for which the reason is not clearly seen.

Here, we are facing a clear antecedent to his 1926 approach: criticizing the division of phase space and looking for periodic or quasi-periodic notions to introduce and justify quantization. Connections between this first attempt by Fermi and the 1926 paper do not end here. Stern's method consisted of using thermodynamic and kinetic analyses of the solid-vapor equilibrium to obtain the aforementioned constant; for the solid, he uses Einstein's model and assumes the existence of a zero point energy. What Fermi proposes in his paper is a new procedure to avoid that hypothesis. Thus, as in 1926, he eludes the quantization of motions with only translational energy and turns to the harmonic oscillator as a system with a recognized and indubitable quantization.

He also introduces an interesting counting system regarding the permutability of atoms. In Fermi's opinion, the atoms of the solid are distinguishable, unlike those of the gas, so the probability of a state with $N_{g}$ atoms of gas and $N_{s}$ atoms of solid must be calculated according to the expression:

$$
P=\frac{N !}{N_{g} !} \int e^{-\frac{W}{\kappa T}} d \omega
$$

where $N$ is the total number of atoms, $W$ the total energy and $d \omega$ the element of phase space, that is defined as follows:

$$
d \omega=h^{3 N_{s}} \prod_{1}^{3 N_{g}} d x_{i} d p_{i} .
$$

Therefore, we can see how Fermi evades the division of the phase space of the gas in cells of magnitude $h^{3 N_{g}}$, but not that of the solid. Fermi stops when he obtains the equilibrium density, and does not calculate the entropy. His method, however, is not as free of weak points as he might claim it is, since precisely the introduction of factorials in (11) conceals a clear distinction between the entropy of the gas and the solid and prevents him from calculating an absolute value for the gas or the solid. Probably for this very reason, and despite the title, Fermi does not end up calculating the entropy constant. 
The other paper on which we must focus our attention, "Considerations on the quantization of systems which contain identical elements" [21], published in Nuovo Cimento in January 1924, presents the idea to which Fermi referred in his seminal 1926 paper [23, p. 187]. The main point is that systems which contain "identical elements" require additional quantum conditions to Sommerfeld rules. Fermi argues that it has been said that it is the impossibility of performing the separation of variables that prevents a correct quantization of the motion of electrons in the case of helium; he believes, to the contrary, that the problem is that it contains two identical electrons, and he proposes to demonstrate how, in the monatomic ideal gas, where separation of variables is possible, a valid expression for entropy can only be obtained if the problem is reduced to that of one particle. To illustrate the peculiarity of these systems, Fermi considers a trio of electrons rotating along the same circle with their positions forming an equilateral triangle. Equivalent rotations are not every $2 \pi$ radians, but every $2 \pi / 3$. This is more or less what Ehrenfest and Trkal had already proposed in 1923 [12] for treating molecules with symmetries. ${ }^{4}$

In an ideal gas, a finite entropy is only obtained if the "gas" is quantized in one way or another. Fermi is thinking about absolute entropy, since neither Boltzmann nor Gibbs ever posed that problem and only considered relative entropies. In any case [21, p. 125]:

In all these hypotheses we will always reduce our system to separate variable systems, which we will quantize with Sommerfeld rules, and we will find, however, that of those hypotheses, the only one that leads to a value of the constant of the entropy in agreement with the experimental value is the one in which the fundamental system, to which our gas will reduce, contains only one atom; while reducing it to systems, even with separate variables, but containing more than one atom, there will always be greater or lesser divergences.

Fermi takes from Imre Brody, who had recently worked in Göttingen, the energy of a particle of mass $m$ in a box [5]:

$$
\omega_{n}=\frac{n^{2} h^{2}}{8 a^{2} m},
$$

where $a$ is the dimension of the edge on which the energy is being studied. Then Fermi calculates the entropy with usual methods, performing sums and avoiding once more the quantization of phase space:

$$
S^{*}=\frac{3 \kappa}{2} \log T=\kappa \log \frac{(2 \pi m \kappa e)^{2 / 3} v_{C}}{h^{3}},
$$

where $v_{C}$ is the volume of a cell $V / N$. The asterisk indicates that this expression is not correct, because there is still one step to go. The probability that there is, as has been assumed, only one particle in every cell must be added. To do this, Fermi calculates the quotient between all the ways $N$ particles can be put in $N$ boxes so that there is only one in each box, and the total possibilities:

$$
P=\frac{N !}{N^{N}} .
$$

Finally, he establishes that the correct entropy is:

$$
S=S^{*}-\kappa \ln P,
$$

and obtains the Sackur-Tetrode equation. To exemplify the appropriateness of his proposal, Fermi shows what would happen if the same is done but with two particles in each box (in this case there would be $N / 2$ boxes). Probability (13) becomes:

$$
\frac{N !}{2^{N / 2}}\left(\frac{N}{2}\right)^{N}
$$

And, in the limiting case where the volume is not divided by any factor, expression (12) remains, which means an extensive expression but does not provide the correct value of the constant. Fermi finishes the paper with a section in which, without calling it by its name, he comments on Gibbs' paradox. Of course, if the treatment he proposes is carried out, a correct entropy of a mixture is obtained: by confining the molecules in cells in which there is only one of each type, he ensures that the entropy obtained is extensive.

${ }^{4}$ Furthermore, some comments and procedures in the previous paper recall the paper by Ehrenfest and Trkal. 
Despite the obvious connection with the previous one, Fermi's paper on Stern's method for calculating the entropy of the ideal gas (which is what he does in this paper) is not even cited. As we have argued, while in the previous one he had been able to calculate the density of the gas through the solid-vapor equilibrium, in the next he found a way to justify the value of the entropy constant. However, the use of factorials and permutations in both cases are not, by any means, in agreement: in the first one the gas differed from the solid in the permutability of its constituents. In his later attempt, the gas molecules are treated, in a certain sense, as nodes of a solid. In any case, we believe that the definition of entropy he uses (14) is illustrative for our purposes. Besides the standard calculation, a factor related to permutations is required. This step is the one he avoided in 1926, because he did not want to make any assumption about the probability of a state.

We have seen that it was Fermi who, rather awkwardly, tried to establish a link between this publication and the 1926 paper. Nonetheless, the only connection is the need to add additional conditions to Sommerfeld rules to deal with identical particles. In no case can this paper be considered a precedent for the exclusion principle, which refers to monoparticular states and not to spatial imaginary cells. But we can learn a lesson: a hierarchy in Fermi's interests. The ideal gas appears as a system in which to test methods to treat multi-electronic atoms.

\subsection{Göttingen and Leiden}

Brody's reference gives us a very good clue of some of the possible consequences of Fermi's stay in Göttingen. As Sebastiani and Cordella have shown, Fermi's sojourn in Göttingen was very productive [46, p. 74]. He prepared and published at least three papers on topics related to quantum theory and issues that interested Born: "The adiabatic principle and systems that do not admit angular coordinates," "Proof that a normal mechanical system is usually quasi-ergodic," and "Some theorems of analytical mechanics that are important for quantum theory" [17-19]. Thus, despite the testimonies that question his interaction with the environment [50, p. 32], the months that the young physicist spent at one of the crucial centers of physics of that time do not seem to have been in vain.

In the early 1920s, Brody worked as Born's assistant and collaborated with him, among other things, on the theory of crystals. In the short paper cited by Fermi [5], he left evidence that he was fully aware of the problem associated with ideal gas quantization, referring to previous works by Sackur [43], Tetrode [59], Planck [41], Stern $[56,57]$. The Hungarian physicist's approach develops a quantum theory of the ideal gas based on Planck's first theory; Paul Scherrer had tried it before, also while at Göttingen, in 1916, but by his own admission, came to the wrong result [47]. Both attempts aimed to amend Planck's proposal based on his second theory, in which quantization had been somewhat diluted [41]. Specifically, Scherrer proposed a way to divide the phase space into cells in order to obtain the entropy constant. Just as previous attempts (Tetrode, Sackur, Keesom and Lenz) did not start from a general formulation, Scherrer draws on the works of Schwarzschild and Debye to quantize quasiperiodic motions. He applied Hamilton-Jacobi's method to the gas particles bouncing off the walls, and obtained an elementary cell smaller than the usual by a factor $2,(h / 2)^{3 N}$, due to a numerical mistake. This led him to a slightly different value of the entropy constant from that of Sackur and Tetrode. However, he emphasized that he had used a quantization that had proven successful in other fields (specific heats and spectra), and downplayed the numerical discrepancy. By the way, with regard to the infamous factor $N$ !, Scherrer's proposal is poor: he introduces it without any comment and gives a hard-to-understand quotation from Gibbs [47, p. 158]. Brody, meanwhile, presented a calculation by simply applying Sommerfeld quantization rules to periodic movements due-as Scherrer did - to the rebounding of free particles in a box. In relation to the controversial dependency on $N$, Brody offered nothing more than the already-typical Planck argument [5, p. 82]:

You have to look at two states as being microscopically not different if they only differ in that two molecules that are completely the same are interchanged...

What we can conclude from all this is that it was doubtless in Göttingen where Fermi became aware, almost firsthand, of the problem of the entropy constant. Brody's paper contains a good sample of many of the papers relevant to that purpose, therefore Fermi was able to learn what had been done up until then, but also that there was a persistent problem with the justification of the factor $N$ ! Scherrer and Brody's contributions have in common with those of Fermi that they aim to find a solid conceptual foundation for the quantization of an ideal gas, and they do not limit themselves to quantizing the phase space, as had previously been done.

In September 1924, Fermi visited Leiden for three months. The choice must have been influenced by his friendship with George E. Uhlenbeck, who had sent him some comments from Ehrenfest on one of Fermi's papers [18]; George W. Ford explains that Ehrenfest was so impressed by this work, produced in Göttingen in April 1923, that he sent a letter to Fermi via Uhlenbeck [26]. The latter worked, in those years, as a tutor to the youngest son of a Dutch ambassador in Rome. He returned to Leiden after three academic years, in 1925, where he became Ehrenfest's assistant. Uhlenbeck's friendship with Fermi undoubtedly prompted the Italian to decide to spend a few months in Leiden. Also in Göttingen, Fermi might have heard of Ehrenfest, though he did not coincide with him. It is commonplace to say that Born, with whom Fermi did work alongside, masterfully taught mathematics during the 
academic year, and in the summer Ehrenfest would go and explain physics; Maria G. Mayer told Kuhn "what a wonderful complement to Born" he was [7, p. 5].

Without a doubt, the scientific conditions in Leiden were very good, if we take into account that Fermi coincided with Einstein just when the famous physicist was finishing his paper on the quantum theory of the ideal gas [40]. It is hard to escape the idea that this might have influenced his subsequent contribution to statistics, despite the fact that it took more than a year until its publication. In a letter to his sister dated October 27, 1924, Fermi wrote $^{5}$ :

As for me, I always feel good and, on average, I make one discovery every twenty days; consequently everyone has a profound respect for me and they feel very honored that I confide in them. Einstein left last week, making enthusiastic declarations of sympathy; in the colloquium, in my honor, German is spoken, since I still find it very difficult to understand Dutch...

\section{And about Ehrenfest:}

Prof. Ehrenfest is a very nice person, although he would not look at all out of place in a second-hand clothes shop in the ghetto; he takes care of the school and its students, and has a special ability to know how to spot talent from the early years from which you can hope for good results for the future.

Ehrenfest was one of the experts in statistical mechanics and was fully aware of the problems of applying quantum hypothesis to ideal gases. We have already mentioned the similitudes between some procedures we find in Fermi's papers and one of Ehrenfest and Trkal [12]. In any event, Fermi did not quote or even mention the Viennese physicist in any of his statistical papers.

In sum, it is evident that Fermi was very up to date with the work and discussions on entropy constant. It may even be that, while in Leiden, he witnessed a first indication of Einstein's quantum theory of ideal gas. However, all of this only gives us the right to frame his contribution of 1926 within these previous interests. None of them anticipates anything like the exclusion principle, and the added rule is not suggested in any of Fermi's earlier work. Of course, his stays in Göttingen and Leiden certainly taught him a very good lesson in the current state of physics, and especially the state of quantum theory and its relationship with the ideal gas.

\section{Toward a new statistics}

We are now going to look at the relation between Fermi's contribution and other proposals from his contemporaries, especially those of Einstein. We will start with the other attempt that Fermi quotes, by Planck. We will see that the father of quantum theory - pace Kuhn — did not properly propose a new statistics.

\subsection{Planck's approach}

Planck published a paper [42] in which he corrected his previous quantum treatment of the ideal gas - questioned as we have seen by Scherrer and Brody - in which quantization was limited to the parceling of the phase space and did not affect the motion of the molecules [41]. Planck thought it necessary to make a drastic modification after Stern and Gerlach's crucial discovery: according to the separation of a beam of silver atoms under the effects of a non-uniform magnetic field, only certain motions can be allowed. Let us emphasize how late Planck - a quarter of a century after presenting his quantum hypothesis - assumed the effective quantization of mechanical motions.

He starts with a standard treatment of the ideal gas in the canonical ensemble, but considering energy quantization. He equals the total number of states of the gas,

$$
z=\frac{1}{h^{3 N}}\left(\frac{e}{N}\right)^{N} V^{N}\left(\frac{4 \pi}{3}\right)^{N}(2 m u)^{3 N / 2}
$$

( $u$ is the monoparticular energy), to the number of states of a particle, $n$, raised to $N$,

$$
n^{N}
$$

${ }^{5}$ Letter from Fermi to Maria Fermi, Leiden, October 27, 1924. Archivo Enrico Fermi. Università di Pisa. 
Hence, the energy of a particle will be quantized as follows:

$$
u_{n}=\frac{h^{2}}{2 m}\left(\frac{3 N}{4 \pi e V}\right)^{2 / 3} n^{2 / 3} .
$$

Within the region delimited by successive $n$ 's, the allowed value is determined through the parameter $\alpha$ that can take values between 0 and 1 :

$$
u_{n}=\frac{h^{2}}{2 m}\left(\frac{3 N}{4 \pi e V}\right)^{2 / 3}(n-\alpha)^{2 / 3} .
$$

For $\alpha=0$, there would exist the maximum value for the zero point energy, and for $\alpha=1$, there would be no such energy. Now, it only remains to do the sums (or integrals) in the usual way but taking into account that parameter, and that is what Planck does. He obtains a new expression for pressure:

$$
p=\frac{N \kappa T}{V}\left(1-\frac{\left(\alpha-\frac{1}{2}\right) h^{3} N}{(2 m \kappa T)^{3 / 2} e V}\right) .
$$

Finally, he checks that his treatment provides an entropy that satisfies Nernst's principle.

In the last section, Planck discusses Einstein's proposal, which eliminates the statistical independence of molecules and implies a "fundamental modification of the usual ideas about the nature and mode of action of the molecules" [42, p. 592]. He closes the paper by comparing Einstein's results with his (based on the parameter $\alpha$ ) in the case of energy per particle. With the experimental measurements carried out so far, it is not possible to decide. $^{6}$

What concerns us here is that Planck does not claim to have made any statistical assumption, or at least not one that differs from the usual treatments. Certainly, the typical argument used nowadays to justify the division by $N$ ! of the partition function of an ideal gas is justifiably attributed to Planck [36, p. 389]. In this paper, he mentions it again, despite the fact that in his new treatment, he does not (or cannot) apply it. Logical, since it is no longer necessary: the particles are occupying, so to speak, volumes $V / N$; hence, the quantities - such as free energy - come out as extensive without any need for correction. As we have seen, this also happened in Fermi's 1924 proposal [21] but with a completely different justification. Planck opposes his idea to Einstein's both for the statistical assumptions and its conceptual implications. Unlike Fermi, in this case the contrast is appropriate, since Planck operates with the usual statistics, which includes statistical independence, but including a numerical parameter that fixes the position of the allowed motions within the cells of the phase space.

\subsection{Fermi vs. Einstein}

There are many differences between Einstein's and Fermi's approaches. Although we have seen that the latter had been interested in the problem of the chemical constant for years, what is most striking in this comparison is the contrast in the presentation of the more general context of the problem. Einstein, at that time, was far from being a neophyte in the matter and it is apparent that he had spent many years mulling over problems that he had run into again and again. The story has been told in detail and precision of how a letter from Bose unblocked the path that led him to formulate the theory of the quantum ideal gas [37, p. 423]. In what follows, we will argue that Fermi did not make use of the path opened up by Einstein, and expressly departed from some of the aspects commented on - and, as a matter of fact, discovered - by his predecessor. All of this is surprising, and it forces us to qualify, once again, the relevance of Fermi's visit to Leiden, just when Einstein was finalizing his theory.

As we have insisted, the main difference in the approach lies in Fermi's clear intention to separate his contribution from any statistical hypothesis. Fermi's statements in this regard are not very clear, and it rather seems that he just wanted to put aside the puzzling statistical peculiarities of the new particles that Ehrenfest - and probably when Fermi was in Leiden - had pointed out to Einstein. When explaining the pros and cons of classical and quantum statistics Einstein argued masterfully that just as in the classical theory one had to choose between obtaining extensive magnitudes and Nernst's principle, in the new statistics both requirements were satisfied at once. Fermi, by dismissing an addition of a $N$ ! factor as irrelevant, showed that he was not aware of these reflections or did not want to enter into these considerations. He preferred to perform the limit of low densities and to identify the temperature with the help of Maxwell-Boltzmann velocity distribution. The Italian physicist argued that in the case of using Boltzmann's principle one must make hypotheses about the probability of the states, and therefore he would not take that route. The method he used in his former paper on "indistinguishable elements" can probably

${ }^{6}$ The expression that Planck uses from Einstein's theory contains a mistake that is also in the original [32, p. 439]. 
give us a clue to interpret that strategy. In that paper, he corrected the entropy through a combinatorial factor (14) related to the permutability of molecules. In his new contribution, Fermi explicitly avoided this procedure.

Needless to say, had he followed that path laid down by Einstein (and Bose), he would have obtained the same result. But he could not have said that adding a factorial of $N$ would not change anything. Let us dwell for a moment on this crucial point. Einstein does use Boltzmann's principle, but he does not use Fermi's Anordnungen (3), but what he calls Verteilungen. The probabilistic assumption that Einstein makes, inspired by Bose, is that the equiprobable event is given by a certain distribution of particles in states, considering as equivalent those states where the number of cells of energy $\epsilon_{s}$ (with degeneracy $g_{s}$ ) with $k$ elements is the same. A state would be fully characterized by the numbers ${ }^{7}$ :

$$
\left\{s_{\circ}^{s}, s_{1}^{s}, s_{2}^{s} \ldots\right\}
$$

where $s_{k}^{s}$ indicates how many cells of energy $\epsilon_{s}$ contain $k$ particles. Then, and again following Bose's reasoning, Einstein argues how to obtain the permutability of a state:

$$
\frac{g_{s} !}{s_{\circ}^{s} ! s_{1}^{s} ! s_{2}^{s} ! \cdots} .
$$

With the usual constraints on energy and number of particles, and

$$
\sum_{k} s_{k}^{s}=g_{s}
$$

he gets the desired result: the expression for $s_{k}^{s}$ that maximizes (16). Then, he calculates the mean occupation number through

$$
\bar{n}_{s}=\sum_{k} s_{k}^{\prime s} k
$$

$\bar{n}_{s}$ is a mean number because it is an average of occupation of all the cells with energy $\epsilon_{s}$ (the prime in $s_{k}^{\prime s}$ indicates the most probable value).

Fermi obtains directly the most probable occupation number without considering the elementary equiprobable event (15). It is in that respect, as we said in the introduction, that he did not take advantage of the breakthrough that Bose's contribution represented. Let us look at it in more detail. If Fermi had followed Einstein's procedure, he would have maximized:

$$
\frac{g_{s} !}{s_{0}^{s !} ! s_{1}^{s} !} \text {. }
$$

If Einstein had followed the Fermi's procedure, he would have maximized:

$$
\frac{\left(g_{s}+n_{s}-1\right) !}{\left(g_{s}-1\right) ! n_{s} !}
$$

because Fermi considered the possibility of distributing $n_{s}$ particles in $g_{s}$ states of energy $\epsilon_{s}(3)$

$$
\frac{g_{s} !}{n_{s} !\left(g_{s}-n_{s}\right) !} \text {. }
$$

The disparity between the two methods lies in the fact that Einstein is considering equiprobable states, elementary events, which remain hidden in Fermi's case. Significantly, Einstein did use the formula (18) in his second paper on the ideal gas, precisely in the discussion that sought to clarify the disagreement of the new statistics with that of Boltzmann's [16, p. 6]. And that is because formulas of the type (18) or (19) (for fermions) are much better suited to a corpuscular interpretation: they express how to calculate the ways to place $n_{s}$ (indistinguishable) particles in $g_{s}$ states. In contrast, formulas of the type (17) and (16) place the emphasis on the cells, the

7 For the sake of clarity, we are not using Einstein's original notation. 
monoparticular states: how many of them are equally occupied. ${ }^{8}$ Strictly speaking, it is not the formula that put the focus on the cells, but the new interpretation - surely unintentional - by Bose, followed and interpreted by Einstein. Boltzmann had used it, but in a different sense. Anyway, Einstein used formulas (18) and (19) to reveal the differences between statistics from a corpuscular point of view, and this shows once again that Fermi either did not know those works very well or he simply did not want to follow Einstein's method. In Einstein's explanation it is crystal-clear that adding a factor $N$ ! to the formula (18), as Fermi proposed, would not be irrelevant if we calculate the entropy from it. And the same happens with (19).

This same connection can be established between Bose's calculation and Planck's calculation for black body radiation. Bose, attempting to stick to Boltzmann's methods as much as possible, started from the equiprobable states of the type (15). In this way, what had been hidden in Planck's formula (18) and later derivations came to light, at least for Einstein. On the contrary, we could say that Fermi's procedure concealed once again what Bose and Einstein had unveiled.

Along the same lines, let us remember that Einstein, doubtless thanks to Ehrenfest's remarks and his own efforts in previous years (decades), was fully aware of the implications of the new theory, which he tried to relate to his now-famous proposal of a dual nature for matter and radiation, which dates back to 1909 [10, p. 117]. Fermi ignores all this, and in fact tries to present his contribution as aseptic, operational, without all the interpretative burden of his illustrious predecessor. In this sense it is revealing to compare the role of the Virial theorem in both contributions. As we have said, this theorem was formulated by Clausius to establish an analogy between mechanical and thermal systems, and its application to ideal gases implies a kinetic interpretation of pressure. Einstein merely stated and underlined that the result obtained coincided with what could be deduced from the theorem. Fermi, by contrast, used it to obtain the equation of state. Einstein obtained the entropy and from there he obtained the thermodynamic properties; Fermi, once he had the kinetic energy, used the Virial theorem to obtain the equation of state, and calculated the entropy starting from its thermodynamic definition (10). Note that from this result, Fermi could have obtained the pressure without going to the Virial theorem, as Einstein had done. But by resorting to Virial theorem he was maintaining the classical conception of the gas, even although Fermi gas presents a pressure so huge that under no circumstances could it be justifiable through impacts against the wall of the container.

The inclusion of the harmonic potential is surely the most upsetting property and what most contrasts Fermi's and Einstein's approaches. The reason why Fermi made use of such a potential is, as he himself made clear, to be able to justify quantization based on Sommerfeld quantization rules. This emphasizes his interest in multielectronic atoms ahead of ideal gases. Thus, it converted the gas into a system with quasi-periodic motions. The years that Einstein had been researching quanta and, especially, the effect of Bose's brilliant idea, enabled him not to justify the quantization of the ideal gas with the periodicity or quasi-periodicity of its motions. He was doubtless aware that in 1924 it was clear that quantization rules left much to be desired, not to say that they had proved invalid [10, p. 300].

In short, we believe Fermi's contribution cannot stand comparison with Einstein's. He avoided all the problematic and intriguing aspects of the new statistics and hardly developed any comment on the puzzling properties of the quantum gas. A poor balance for a foundational paper.

\subsection{From the old to the new}

Despite having focused on Fermi's contribution, it seems that a brief comment on the immediate contributions of Dirac and Heisenberg on the problem of many body [9,29] is essential to complete the picture, especially inasmuch as they will allow us to outline and underline some of the ideas that we want to emphasize.

Fermi presented his paper at the Academia dei Lincei in February 1926, and the editors of Zeitschrift für Physik received the German version at the end of March. It was published in the May issue, a month before Heisenberg sent his manuscript, and three months before Dirac sent his to the Proceedings of the Royal Society. Fermi's paper is not cited in any of those articles, though Dirac confessed having read it without giving it much importance ${ }^{9}$ :

Dirac: I had read Fermi's paper about the Fermi statistics and forgotten it completely.

Kuhn: Had you?

Dirac: Absolutely forgotten it, and when I wrote up my work on the antisymmetrical wave functions, I just didn't refer to it at all because I had completely forgotten it. Then Fermi wrote and told me, and I remembered that I had previously read about it.

Indeed according to Belloni [1, p. 107], Orso M. Corbino, Fermi's academic godfather in Italy, recommended to him - if not compelled - to contact Dirac [34, p. 36]:

8 In modern terms we would say how many of these monoparticular states are involved in the global wave function. In [49, p. 362] we discuss these points in a more general context.

9 American Institute of Physics, Oral Histories, P.A.M. Dirac-Session III, May 7, 1963. 
In your interesting paper "On the theory of Quantum Mechanics"... you have put forward a theory of the Ideal Gas based on Pauli exclusion principle. Now a theory of the ideal gas that is practical identical to yours was published by me at the beginning of $1926 \ldots$ Since I suppose you have not seen my paper, I beg to attract your attention on it.

Dirac recalled years later: "I saw it was the right statistics for electrons in an atom, but I didn't feel that it had wider applications." As a matter of fact, Dirac understood Fermi's intentions quite well: as we maintain, Fermi never posed his result as a fundamental part of the new mechanics. Although in the letter in which he claimed his authorship he describes Dirac's result — and his own — as an ideal gas theory based on Pauli exclusion principle, this is not what Dirac did. He constructed two quantum statistics by imposing the non-observability of the difference between identical particles. That is, he began by establishing that the state associated with a system of equivalent elements, such as the electrons of an atom, should not distinguish permutations between them [9, p. 662]:

If the positions of two of the electrons are interchanged, the new state of the atom is physically indistinguishable from the original one. In such a case one would expect only symmetrical functions of the co-ordinates of all the electrons to be capable of being represented by matrices. It is found that this allows one to obtain two solutions of the problem satisfying all the necessary conditions, and the theory is incapable of deciding which is the correct one. One of the solutions leads to Pauli's principle that not more than one electron can be in any given orbit, and the other, when applied to the analogous problem of the ideal gas, leads to the Einstein-Bose statistical mechanics.

Thus, it is not just a question of applying Pauli principle, but rather of deducing it. Dirac insists on the property on which he will build both statistics [9, p. 667]:

Consider now a system that contains two or more similar particles, say, for definiteness, an atom with two electrons. Denote by $(m n)$ that the state of the atom in which one electron is in an orbit labelled $m$, and the other in the orbit $n$. The question arises whether the two states $(m n)$ and $(n m)$, which are physically indistinguishable as they differ only by the interchange of the two electrons, are to be counted as two different states or as only one state, i.e., do they give rise to two rows and columns in the matrices or to only one? If the first alternative is right, then the theory would enable one to calculate the intensities due to the two transitions $(m n) \rightarrow\left(m^{\prime} n^{\prime}\right)$ and $(m n) \rightarrow\left(n^{\prime} m^{\prime}\right)$ separately, as the amplitude corresponding to either would be given by a definite element in the matrix representing the total polarization. The two transitions are, however, physically indistinguishable, and only the sum of the intensities for the two together could be determined experimentally. Hence, in order to keep the essential characteristic of the theory that it shall enable one to calculate only observable quantities, one must adopt the second alternative that $(\mathrm{mn})$ and $(\mathrm{nm})$ count as only one state.

The new approach is masterfully explained and there is no room for doubt about Dirac's intentions and interpretations. In the entire paper he always refers to individual particles, as is logical given the symmetrization method based on particle indexes; but we can also find expressions of the type "more than one molecule can be associated with each wave" [9, p. 672]. Dirac clearly opts for associating Bose-Einstein statistics (symmetric functions) to lightquanta, and the new statistics to matter; that is why he develops some of its properties and points out that it does not imply the condensation so characteristic of the other one. Dirac was fully aware of Einstein's contributions and methods.

In the case of Heisenberg, the other physicist who laid the quantum-mechanical basis for dealing with many body systems (and helium problem), things occurred differently, both formally and in connection with Fermi's contribution. According to Heisenberg, on a trip, he made with a friend to Italy in the spring of 1926, he happened to visit Fermi, whom he had met in Göttingen in $1923 .{ }^{10}$. He also remembered that Fermi did not seem to be very comfortable in the German city during his stay in 1923. On the contrary, in their meeting in 1926, when Fermi was probably involved in drafting his paper, they had a very pleasant discussion. Heisenberg does not seem to be very clear about what happened, since he gives a description of what Fermi told him that strongly recalls his own contribution [29] rather than that of the Italian's. He emphasizes the influence of that conversation which, in fact, he never had mentioned before ${ }^{11}$ :

He told me that when one applied Pauli's exclusion principle one got something which is somewhat related to the Bose statistics, but which is definitely different from Bose statistics. It was a kind of complement. He told me that the relation between his statistics and Bose's was something like plus and minus. So that I knew from Fermi. The paper had not appeared at that time. It must have been April of '26 and that certainly must have influenced my paper on this resonance business... Do I not quote my conversation with Fermi?

\footnotetext{
10 American Institute of Physics, Oral Histories, Interview of Thomas S. Kuhn to W. Heisenberg-Session V, 15 February 1963.

11 American Institute of Physics, Oral Histories, W. Heisenberg-Session IX, 27 February 1963.
} 
In his contribution Heisenberg claims to have found a connection between Bose-Einstein statistics and the Pauli principle. In the aforementioned interviews, Heisenberg did not hide the fact that in his first paper he had barely understood the point, unlike Dirac. His interest, he claims, lay in helium and the two-electron system. When he saw that his treatment could be applied to gases and general systems with $N$ equivalent particles, he remembered what he had discussed with Fermi, and attempted to draft his contribution. Needless to say, it opened up the way to definitively solving the problem of ortho and parahelium.

Heisenberg's treatment begins with general considerations about the corpuscular and wave views, since it is surely one of his first publications after the publication of Schrödinger's wave mechanics. Just as he celebrates the equivalence of matrix mechanics (quantum, for him) and wave mechanics, he dismisses the wave view as inadequate. Thus, despite the fact that there is no longer any doubt that the space- time preconceptions of kinematics will have to be changed, Heisenberg clings to corpuscular vision. He considers the problem of two particles in detail, and studies the breakdown of the degeneration caused by the Coulombian interaction; degeneration only remains when the particles are not in the same monoparticular state. The solutions of a system like this belong to two exclusive families, the symmetric and the antisymmetric. This is how Heisenberg lays the groundwork for identifying the two spectral series of helium. He proposes interpretations of indistinguishability by appealing to de Broglie wave properties, but he also imagines particles constantly exchanging their positions [29, p. 421, pp. 425-426].

Neither Dirac nor Heisenberg discussed in detail the link between the new discoveries with the old Boltzmann counting system. Fowler did so, and in a paper that we will see in the next section, he proposed a general framework that encompasses the three statistics. Ehrenfest and Uhlenbeck also devoted a short paper to this question in December 1926 [14], the new statistics fresh out of the oven. In this short note, they analyzed a one-dimensional ideal gas, whose Schrödinger equation is:

$$
\left[\frac{\partial^{2}}{\partial x_{1}^{2}}+\frac{\partial^{2}}{\partial x_{2}^{2}}+\cdots+\frac{\partial^{2}}{\partial x_{N}^{2}}+\frac{8 \pi^{2} m}{h^{2}} E\right] \psi=0
$$

( $x_{i}$ is the coordinate of particle $i$ and $E$ the total energy). Solutions will be of the form:

$$
\psi=\sin k_{1} x_{1} \sin k_{2} x_{2} \cdots \sin k_{N} x_{N},
$$

where $k_{i}$ are whole numbers that can be the same or different. Energy will be:

$$
E=\epsilon_{k_{1}}+\epsilon_{k_{2}}+\cdots+\epsilon_{k_{N}}=\sum_{s} n_{s} \epsilon_{s}
$$

where $n_{s}$ gives the number of times that the value $k_{s}$ appear in the wave function. Monoparticular energies are given by:

$$
\epsilon_{s}=\frac{h^{2}}{8 \pi^{2} m} s^{2}
$$

with $s$ a whole positive number. The number of solutions (20) with the same energy can be obtained through:

$$
\frac{N !}{n_{1} ! n_{2} ! \cdots}
$$

According to Heisenberg, antisymmetric solutions built by starting from determinants would be the only valid ones, so that each value of $k$ appears at most once in (20). According to Dirac, there would be two possibilities, symmetric and antisymmetric functions, and would correspond to Bose-Einstein or Pauli-Fermi-Dirac statistics (sic). The solutions (21) constructed starting from permutations of the $k$ 's in (20) would be the proper solutions of Boltzmann statistics. ${ }^{12}$

We are interested in highlighting two points in this note. On the one hand, Ehrenfest and Uhlenbeck do not mention the problem of $N$ ! or the entropy constant, in line with what Ehrenfest had previously defended [12]. On the other, we find, in the footnote that closes the paper, an interesting statement on the incompatibility of the wave view with the idea of particle:

For $N=2$ or 3 the geometric comparison of $\psi, \psi^{\text {symm }}$ and $\psi^{\text {antisymm }}$ is very illustrative. But how do these $N$-dimensional wave motions have to be imagined into one-dimensional wave motions, or completely into the translational motions of the molecules of our one- dimensional gas? This question of illustration seems to us

12 Elsewhere we have proposed an alternative deduction of Boltzmann's statistics starting from wave mechanics [48]. 
to be particularly important for understanding Pauli's prohibition. While Pauli's prohibition in its original form (prohibition of identical quantum motions of electrons in the atom) was so illustrative, as far as we know there is no corpuscular interpretation for the extended Pauli prohibition (only permit $\psi^{\text {antisymm }}$ ).

Hence, they were not at all satisfied by the mere statistical description (usual nowadays) of Pauli principle: there cannot be two particles in the same state.

Let us now see what other contemporaries said about this point, especially those who undertook the first applications and developments of Fermi statistics. Later on, to conclude, we will poke our noses into the two famous conferences on physics in 1927: the commemoration of the second centenary of the death of Volta, held in Como in September, and the fifth Solvay Conference, held in Brussels in October. All these forays will help us to evaluate how Fermi's proposal was understood, once again paying special attention to the indistinguishability of molecules.

\section{The statistics for material particles}

We will begin by taking a look at some papers by Fowler. He was an expert in statistical mechanics, the supervisor to Dirac's thesis in that same year of 1926, and he was responsible for directing the young Dirac's attention to Heisenberg's work on the new mechanics. Hence, it should come as no surprise that he was very much on the case with regard to Dirac's contributions to quantum statistics. We will continue with a crucial paper by Pauli in which he used the new statistics to explain a magnetic property of metals, until then difficult to understand. This application led to a second one, by Sommerfeld, generally better known than Pauli's, since it undoubtedly refers to the most common application of Fermi statistics: electron gas. As we will see, despite the fact that the new language and approach of quantum mechanics was already emerging, all of these developments fit together better as the last developments of the old quantum theory.

\subsection{Fermions in the stars}

At the end of 1926, Fowler published two papers [27,28] where he clearly opted for the Fermi-Dirac statistics as the correct one for material particles, and consequently Bose-Einstein's for radiation, as became common in those initial steps of quantum statistics. In the first paper, "General forms of statistical mechanics with special reference to the requirements of the new quantum statistics," quantum mechanics hardly makes an appearance. It is worth mentioning that in the acknowledgments, Fowler records the discussions held on this topic during his stay at the Institute for Theoretical Physics in Copenhagen, and especially those that he held with Bohr. Hence, we can take Fowler's publication as a good sample of the state of affairs in a wider circle than Cambridge.

The main novelty, at least as Fowler presents it, is "to define the entropy (and the absolute temperature) in strict analogy with rational thermodynamics by means of the equation $d Q=T d S^{\prime \prime}$ [27, pp. 432-433]. In other words, what the author aims to do is to proceed in a strict sense from the point of view of the fundamentals of statistical mechanics: to obtain statistical functions starting from mechanics and then equate them, by analogy, to thermodynamic quantities. And to do this without being restricted to a specific system, thus the ideal gas would be an application of a more generic and grounded approach. Needless to say, Fowler would proceed by using the method known today as Darwin-Fowler method. As we have seen, Einstein's treatment, unlike Fermi's, could be considered more grounded from a thermodynamic point of view. But Fowler aims to extend those foundations and to focus a little more on the statistics that in his opinion corresponds to material particles: that of Fermi-Dirac.

Regarding the issue of identity of particles and its new status in the new statistics, Fowler restricts the new meaning of "identical" to "having the same set of energy" [27, p. 434, footnote]. That is, what characterizes a system is no longer its position or moment but its spectrum. ${ }^{13}$ But this does not at all mean that the concept of particle is weakening. We find it in the seventh section, focused on the specific case of the ideal gas. Fowler writes [27, pp. 440-441]:

In discussing moving mass points, it is impossible to admit that we can class together all mass-points moving with the same energy as indistinguishable systems. It must be possible ideally to recognize differences between particles moving with the same energy in different directions.

And he continues:

13 In close connection with this, elsewhere we have discussed the difference between state statistics in contrast to particle statistics [49, p. 356]. 
In Einstein's form of statistics the simplification made by Einstein in his original paper cannot therefore be completely justified, but as would be expected, it is without effect on the result, which can be reached without it.

Fowler is referring to the identity of the particles with the same energy, but moving in different directions. Although he criticizes this "simplification made by Einstein," (referring, we guess, to his combinatorial treatment) he acknowledges that it does not affect the final result: Einstein took into consideration the degeneration of classical energy and limited the quantization to "cutting up the phase space of a single molecule into cells of extension $h^{3}$ " $[27$, p. 442]. It was not until the new statistics could be formulated in terms of Fock's space that those questions were fully clarified. Before, with the concept of a particle still fully in force, they remained somewhat obscure.

In the following sections Fowler applies the new statistics to crystals and briefly comments that the calculation of fluctuations does not vary substantially (although the result does, as Einstein showed in 1925, and about which Fowler says nothing). It is in the tenth section, devoted to arguing that each of the quantum statistics should be used respectively in contexts of radiation and matter, where Fowler refuses to admit two photons with the same energy as identical [27, p. 444]:

Just as for atoms it is logically incorrect and unnecessary to consider quanta of equal energy (frequency) but moving in different directions as indistinguishable, or quanta of equal energy and direction but different polarization. The final result is unaffected.

Consequently, Fowler argues against Einstein, who proposed applying the new statistics to matter. According to Fowler it is plausible to think that statistics for matter and radiation are different, since in the first case atoms are conserved and in the second photons are not. He suggests a link between Pauli principle and impenetrability, or impossibility of superposition of matter. ${ }^{14}$ Once again we witness the validity of the corpuscular or visual conception of Pauli principle. In fact, Fowler returns again to the topic of corpuscularity, calculating spatial distribution of particles. In the case of the absence of external potentials, he presents a calculation that corresponds to the classical one, since the approximation he makes is the only one that specifically allows him to obtain some spatial distribution. As is known, in quantum treatments an analytical relation between the number of particles $N$ and the chemical potential $\mu$ for an ideal gas does not exist if we keep within the range where Maxwell-Boltzmann distribution is no longer valid. This can only be done by performing the limit, which is equivalent to losing the characteristic properties of quantum statistics. That is, when:

$$
N=\frac{(2 \pi m \kappa T)^{3 / 2}}{h^{3}} V \mu
$$

Hence, Fowler's argumentation on spatial distribution is only pertinent in the classical domain.

The second paper by Fowler tackles the first application of Fermi statistics to a physical system, stellar matter [28]. Based on Eddington's research and speculations on the constitution of stars, Fowler proposes to solve one of the paradoxes that the famous English astronomer had encountered. The rate of emitted radiation was impossible to explain with a gas of ionized particles regulated by classical statistics. Fowler proposes to undo the paradox with the help of the new relation between energy and temperature obtained through the Fermi-Dirac statistics. Once again we find evidence that the corpuscular conception was fully in force. This is what Fowler wrote on Pauli principle [28, p. 115]: "Its essential feature is a principle of exclusion which prevents two mass-points ever occupying exactly the same cell of extension $h^{3}$ of the six-dimensional phase-space of the mass-points."

In the paper we find an argued explanation of today's typical result about the huge energy a Fermion gas has at zero temperature. At the end of the paper, Fowler speculates that the enormous repulsion caused by the great pressure of a gas of these characteristics is due only to the "quantum constraints" [28, p. 122]. That is, the enormous repulsion that has been estimated between electrons is due, not to some type of interaction or collision, but to the "quasi-thermodynamic consequences of the existence of the quantum constraints embodied in Pauli principle." Something that Fermi had not pointed out. It is worth recalling here that Einstein, after having found the quantum theory of the ideal gas, looked in other directions to explain the decrease in pressure relative to an ideal Bosonic gas. This led him to the de Broglie hypothesis, but also to conjecture some kind of interaction [40]. Fowler proposes that it is simply the quantum prescription itself that implies such a significant increase in the work necessary to compress the volume of a gas that follows the Fermi statistics.

Before moving on to the next development, we would like to mention one of the applications of the new statistics made by Fermi himself [24]: the Thomas-Fermi model, so named because a year earlier, in December 1926, the English physicist Thomas proposed basically the same idea [60]. Thomas thanked Bohr and Kramers for their help while he was performing the calculations in March 1926, when he was in Copenhagen. So he probably developed his model with Pauli principle in mind but not the new statistics. This application consists of a rough calculation of the distribution of electrons in heavy atoms, based on general assumptions and without trying to make exact

14 Ehrenfest proposed a similar idea, but later dropped it [13]. 
calculations. It makes it possible to estimate ionization energies and electronic levels to account for the periodic table. Both Thomas and (a year later) Fermi, who did not know about Thomas's contribution, included, among their hypotheses, that electrons satisfied the exclusion principle. Fermi also made use of the magnitudes calculated in his paper on ideal gases. However, and in relation to the more fundamental questions that interest us here, such as the status of the identity and corpuscularity of electrons, these publications did not contribute much: in both papers, the classical visualization of electrons is still perfectly prevailing and, in fact, the authors are attempting to get an idea of their spatial distribution in the atom.

\subsection{Pauli and Sommerfeld on metals}

In a letter written to Rasetti almost thirty years after the publication, we are going to discuss now [39], Pauli states $^{15}$ :

I was so glad that I had eventually an answer to the question $<<$ If it's true, that the electron has a spin, why then there is not a strong paramagnetism in metals according to the Curie-law? $>>$. This question was raised by many physicists, particularly by $\underline{\mathrm{O} \text {. Stern. }}$.

Indeed, Pauli saw in Fermi's contribution an opportunity to account for some of the properties of metals that could not be explained by current theories, in particular their limited contribution to paramagnetism. He was aware of Dirac's contribution:

Incidentally, the paper of Dirac (...) is independent of Fermi's paper. Dirac was in Copenhagen in the autumn of 1926 and I wrote to him, there, whether he knows how a spin of the atoms (or electrons) would modify the results. I also mentioned Fermi's paper. He answered me, that he never considered this question and that Fermi's paper was entirely new to him. Immediately after that I started to work on this question myself (autumn 1926), and I found very quickly all answers.

Which supports Dirac's version that he had barely noticed Fermi's paper. In general terms, Pauli's contribution contains the first treatment of the two statistics with the approach of the grandcanonical ensemble, the method that Kramers would take up years later [35]. Pauli begins by commenting on the state of the art of degenerate gases. He knows and cites the contributions of Bose, Einstein, Fermi, Heisenberg and Dirac, and takes from the latter the idea that Fermi-Dirac statistics are the most suitable for electrons, since they have produced good results with helium. As we see, this attribution to each statistics (matter vs. radiation) was becoming gradually established. Pauli takes advantage of this introduction to refute from the outset a possible criticism of the validity of Fermi statistics: Einstein's arguments in favor of the de Broglie hypothesis, supported by Schrödinger's treatment, do not apply, at least immediately, to the new procedure. Even so, Pauli recalls that the matter-radiation analogy has its limitations, especially when dealing - as this paper does - with systems with many bodies. Waves, in this case, are defined in a multidimensional space, unlike radiation, which can always be described in well-known three-dimensional space. However, he emphasizes his intention to maintain the corpuscular and undulatory interpretations on an equal footing [39, p. 82]:

... we want to understand the corpuscular and the wave-like representation of the theory as two equal descriptions of the statistical behavior of the gas, without giving preference to one over the other.

Pauli develops an extension of what causes the absence of total angular momentum of the two electrons in helium even though they have intrinsic magnetic momentum. Thus, he tries to explain the magnetic behavior of some metals, treating their electrons as an ideal gas in the first approximation. However, before entering into the matter, Pauli elaborates a very complete development of the two statistics with a new ensemble created for this purpose. As a matter of fact, it is the grandcanonical, although Pauli does not refer to chemical potential. ${ }^{16} \mathrm{He}$ presents his procedure through an analogy with the transition from microcanonical to canonical ensemble, which culminates in the calculation of fluctuations of the mean number of particles in each state $s\left(n_{s}\right)$,

$$
\overline{\Delta_{s}^{2}}=\overline{n_{s}^{2}}-\left(\overline{n_{s}}\right)^{2} .
$$

If $Z$ indicates the number of states with energy $\epsilon_{s}, \overline{N_{z}}=Z \bar{n}_{s}$, and $\overline{\Delta_{z}^{2}}=Z \overline{\Delta_{s}^{2}}$, and then:

$$
\overline{\left(\frac{\Delta_{z}}{\overline{N_{z}}}\right)^{2}}=\frac{1}{\overline{N_{z}}} \pm \frac{1}{Z},
$$

\footnotetext{
15 Pauli to Rasetti, October 6, 1956. In AHQP, microfilm 66, Sect. 12.

16 Pauli does not bother to identify the new parameter that he needs to break free from the constrain of a fixed $N$. As far as we know, until then, Gibbs grandcanonical ensemble had only been used for (classical) gas mixtures.
} 
expression previously calculated by Einstein (with the sign + ) but not by Fermi. Sign + corresponds to Einstein statistics, and - to Fermi's. Pauli uses the concept of particle, but with relevant nuances:

Perhaps this behavior can be interpreted by assuming phase relations between the different de Broglie proper vibrations, and thus give a hint for a future physical explanation of the basic assumption of Fermi statistics and thus also the "equivalence rule."

"Equivalence rule" was what the exclusion principle was known as at that time. The basic idea expressed by Pauli is that instead of questioning the individuality of the particles, he, as Einstein did two years earlier, attributes a certain type of interaction to them which results in a statistical dependence. In what follows, Pauli extends what he obtained to gases with magnetic moment, in which the equivalence principle would also govern, and finally accomplishes his objective: calculating magnetizations and susceptibilities for zero temperature, obtaining qualitatively correct results. He does not expect to go much further, given the simplistic model (ideal gas).

When Sommerfeld learned about Pauli's work he encouraged him to develop it and to obtain more properties of metals that the Drude model could not explain. Pauli never got round to doing it, probably because he was focused on developing other more fundamental aspects of the new mechanics [11]. It was Sommerfeld who took up the matter, as Pauli explained to Rasetti in the letter we have already quoted ${ }^{17}$ :

Sommerfeld passed Hamburg in the spring of 1927, where I gave him proofs of my paper. The next day he said to me, that he was very much impressed by it and that one should make further applications to other parts of metal-theory like Wiedemann-Franz law, thermoelectric effects, etc. As I was not eager to do that, he made then this further applications himself.

Sommerfeld presented a schematic version of his ideas in October 1927 in Die Naturwissenschaften [52], and did not publish an extended version until the following year, in Zeitschrift für Physik [54]. ${ }^{18}$ Before that, he presented some of his results at the Como conference, in Italy, in early September 1927 [55]. There, Sommerfeld was very clear about the novelties introduced by the new statistics. He reduced them to two: The way of counting equiprobable states and the dimensions of the elementary cell $h$.

He identified Einstein statistics with Schrödinger's wave mechanics, since the latter had redone the calculations starting from the de Broglie hypothesis mentioned by the former. Sommerfeld introduced Fermi statistics in the same frame, emphasizing that at low temperatures (which includes room temperature) practically all properties are independent of temperature. In a subsequent installment in Die Naturwissenschaften [53], Sommerfeld described electrons with low quantum numbers as a "Volk ohne Raum," a political metaphor for Germany's situation in those years. He again underlined the new conception of electrons from Schrödinger's wave mechanics, and pointed out that they must be understood more as states than as individuals. However, this does not imply that Sommerfeld stopped referring to electrons as particles in what follows. In short, Sommerfeld did not dwell too much on interpretative issues of the new statistics, in accordance with what he did in relation to other fundamental matters of the new mechanics. Despite his clear preference for Schrödinger's formulation over Heisenberg's, he did not completely abandon the idea of particle or try to substantiate the new way of counting. He merely sought applications and solved old problems in the theory of metals.

We completely agree with Hoddeson et al. [30]: the Fermi-Pauli-Sommerfeld theory is a semi-classical theory, a quantum refinement of Drude's theory, and a typical product of the old quantum theory. In fact, Sommerfeld's theory explains many things, while failing to explain many others. It would not be until Felix Bloch, in 1928, working in the recently created Leipzig group with Heisenberg, formulated his quantum theory of solids, that we can properly speak of a quantum mechanical theory of electrical conduction. Bloch explains, for example, why the mean free path of electrons is much greater than the interatomic distance, a weak point in Sommerfeld's approach. Bloch's theory of metallic electrons was to that of Pauli-Sommerfeld what Dirac's statistics was to that of Fermi.

\subsection{Como and Brussels}

At the Como Conference [6], Fermi became a celebrity in his own country and in a way, another member of the international community of physicists. According to Rasetti, it was there that Fermi and Pauli met for the first time [25, p. 179]. Pauli recalled that Heisenberg introduced them by saying something along the lines of "May I introduce the applications of the exclusion principle to each other." In the discussion that followed Sommerfeld's presentation, Lorentz raised some objections related to the application of the exclusion principle. What would happen if two pieces of metal were joined with an electron in the same state? Fermi replied that the new allowed states had to be recalculated, but that under no circumstances would we find two electrons in the same state in the new metal. Planck then correctly deduced that quantization did not apply to electrons but to the gas as a whole.

17 Pauli to Rasetti, October 6, 1956. In AHQP, microfilm 66, Sect. 12.

18 In the same issue papers on specific applications by his assistants, Houston and Eckart, were published [11]. 
In this discussion, Fermi stated that he himself had been working on applications of his statistics to properties of metals, such as energy of vaporization, but that he had only obtained qualitative results. For instance, he verified that the order of magnitude of some quantities was in good correspondence with the measurement. According to Rasetti [25, pp. 179-180], Corbino was certainly studying specific heats of metals at low temperatures in Rome, both theoretically and experimentally, and he suggests that Fermi was already aware of the main application of his statistics even before Sommerfeld's works.

Fermi also took part in the discussion that followed Bohr's famous speech in which he presented his principle of complementarity. We find once again a significant allusion to the role of statistics. He differentiates his approach from Einstein's because in the latter there is a lack of statistical independence, and in his own treatment only Pauli principle is used [25, p. 180]:

To overcome these difficulties, two attempts have recently been made, one by Einstein and one by me: in Einstein's case, a statistical dependence of the type proposed by Bose for the quanta of light is admitted among the gas molecules; in my case, the Pauli exclusion principle was applied to the gas as a whole, considered as a single system made up of all the molecules (which are identical to each other).

This quote, made with the necessary time for reflection, and after becoming aware of Heisenberg's and Dirac's contributions, would be enough to show that Fermi did not see in the indistinguishability of the particles a distinctive characteristic of his approach. Let us see how he considers those contributions [25, pp. 180-181]:

The relationships between these two statistics have been clarified by means of the new mechanics from the works of Heisenberg, Dirac and Wi[gner]: they have shown that if you have a system that contains identical particles among them, their terms are divided into groups so that it is not possible in any way to obtain transitions between two terms belonging to different groups. One of these groups satisfies Bose-Einstein's statistics and the other satisfies Pauli principle of exclusion, and therefore the statistics proposed by the author. Experience has shown so far that the electrons of an atom and even the positive corpuscles always satisfy the principle of exclusion.

Hence, Fermi once again does not emphasize indistinguishability of molecules at all. ${ }^{19}$ Bohr, in the final part of his presentation, referred to Heisenberg's contribution on resonance. There, we do appreciate a forecast of the loss of individuality of the electrons according to complementarity. He suggests that it would be necessary to delve into a field theory that accounts for the results obtained so far for electrons and for quanta of light. Heisenberg stated in the discussion that the protons also followed Fermi statistics, starting from the analogy between the electrons of helium and the nuclei of the hydrogen molecule.

Some of these topics were taken up the following month at the famous fifth Solvay Conference. At that meeting, in collaboration with Born, Heisenberg devoted part of his presentation to quantum statistics. Fermi did not attend the conference, despite the fact that Einstein proposed him to Lorentz as his own substitute to discuss the new statistics, in one of the first times that Einstein admitted (privately) that he did not agree with the new developments ${ }^{20}$ :

I recall having committed myself to you to give a report on quantum statistics at the Solvay congress. After much reflection back and forth, I come to the conviction that I am not competent [to give] such a report in a way that really corresponds to the state of things. The reason is that I have not been able to participate as intensively in the modern development of the quantum theory as would be necessary for this purpose. This is part because I have on the whole too little receptive talent for fully following the stormy developments, in part also because I do not approve of the purely statistical way of thinking on which the new theories are founded... Up until now, I kept hoping to be able to contribute something of value in Brussels; I have now given up that hope. I beg you not to be angry with me because of that; I did not take this lightly but tried with all my strength... Perhaps Herr Fermi in Bologna ${ }^{21} \ldots$ or Langevin... could do a good job.

Heisenberg and Born did discuss quantum statistics in terms of the resonance between identical elements. They associate, as usual, Bose-Einstein with quanta of light and Fermi-Dirac with electrons and - no doubt-protons. However, they believe that for neutral particles the pertinent statistics is that of Bose. While they do not develop this point, they mention an alternative approach by Jordan, based on an analogy between photons and electrons, who in this way would have also arrived at the Fermi-Dirac statistics on his own.

In the general discussion of the conference, the issue of the scope of application of each statistics came up again. It seems that Langevin was in charge of making a very brief previous presentation on quantum statistics (his was another of the names Einstein suggested to Lorentz). The recommendation seems to have been correct: Langevin is unambiguous about the change - if we might put it thus - of paradigm [51, pp. 268-269]:

19 The work by Wigner that he quotes is [61].

20 Einstein to Lorentz, June 17, 1927. In [37, pp. 431-432].

21 Fermi was not in Bologna. 
Il semble aujourd'hui qu'on doive modifier cette manière de faire en supprimant l'individualité des constituants du système et en y substituant l'individualité des états de mouvement. En supposant qu'un nombre quelconque de constituants du système peuvent avoir le même état de mouvement on obtient la statistique de BoseEinstein.

Thus, the individuality of the particles must be replaced by the individuality of the states. In the discussion, Heisenberg once again insisted that the new mechanics did not in themselves provide arguments in favor of one statistics or another, and insisted that the interaction between the elements had nothing to do with the way of counting (we assume it was referring, for example, to the Coulombian interaction). Kramers again referred to Jordan's analogy between electrons and photons, but Dirac criticizes Jordan's proof, in which, he claims, there were artificial procedures and mathematical errors. Dirac states that a wave treatment only leads to Bose-Einstein statistics.

In short, at Solvay the new statistics were discussed, both in the presentation by Born and Heisenberg and in Langevin's final discussion. The issue of the greatest concern was the scope of each of them, and among those present, Heisenberg seems to be the most knowledgeable on this issue. We would like to emphasize that, with regard to conceptual clarity, Langevin's brief exposition appears to us to be extremely accurate in relation to the change of perspective introduced by the recent developments: the leading role had jumped from particles to states.

\section{Final remarks}

A critical reading of Fermi's seminal paper reveals that it is far from being a contribution in which the implications of a the new statistics are fully discussed. Rather, it consists of an exercise of the application of Pauli principle to gas theory that expressly avoids going deeper into statistical and conceptual questions. Both in the paper and in the statements that immediately followed, Fermi did not deal with the allegedly new status of particles. He presented and defended a pragmatic statistical treatment, which is perfectly illustrated by the way he introduced temperature in his 1926 paper. A few months later, Fowler tried to provide a more solid foundation, in addition to tackling some conceptual challenges, as how to understand the huge pressure of a Fermi gas.

Accordingly, it is clear that Fermi did not at all follow the framework of what Einstein had previously done. The differences are many and diverse. The most striking one is the lack of reflection on the new result. Perhaps Fermi's youth can explain this contrast with Einstein's seminal works. The role of quantization illustrates that gulf quite well. In Einstein's theory, the quantization of energy did not play a central role and, following the path opened up by Bose, he focused on the structure of phase space and on the way states were counted. Fermi was not completely detached from the original quantization of energy by Planck, to the point of resorting to a harmonic potential that allowed him to use the energy levels of the harmonic oscillator. Einstein's papers contain developments and reflections that had practically matured during twenty years of reflection. None of that can be found in Fermi's contribution. The influence that these papers of Einstein's had on Schrödinger, for example, also illustrates how rich they were in terms not only of presenting a new statistics, but in comments, analysis and hypotheses. We do not find any of that in Fermi's paper. Although this does not justify the scarcity of historiographical studies that have been devoted to Fermi statistics, it undoubtedly makes it a less attractive route to quantum statistics, both from a physical and a historiographical point of view.

Hence, contrary to what has been noted on some occasions, it does not seem that Fermi's stay in Leiden was particularly influential in this regard. Let us remember once again that Fermi was there when Einstein was in Ehrenfests' place finalizing the second installment of his quantum theory of the ideal gas. But none of the issues dealt with in that paper, with which both Einstein and Ehrenfest were very much concerned, show up in Fermi's contribution. On the contrary, it could be deduced (though not with total certainty) that Fermi had become familiar with the problem of ideal gases the previous year, when he visited Göttingen and worked alongside Born and his assistants, such as Brody. We have also seen how his contact with Heisenberg even led the creator of quantum mechanics to publish his work on resonance and quantum statistics after a visit to Rome in 1926. So it could even be said that the stay in Göttingen was more profitable than the one in Leiden.

Fermi did try to establish a link between his 1926 paper and some of his earlier contributions to the problem of the gas constant. However, the link is far from being straightforward, except for demonstrating a prior interest in the subject. That interest prepared him to immediately detect the new possibilities that the exclusion principle opened. The study of Fermi's early papers has allowed us to understand a little better what Fermi meant by avoiding (in 1926) statistical hypotheses. On the other hand, and contrary to what has been said (and is attributed to Fermi), we have argued that nothing indicates that the Italian physicist was about to find the exclusion principle on his own ([50], p. $36 ;[46]$, p. 91$)$ :

Fermi was gropping for something that eluded him. We now know this was Pauli's exclusion principle, but falling short of this discovery, which was to be the key to so much of physics, Fermi was unknowingly preparing for its application to statistical mechanics. 
None of this can be inferred from Fermi's previous work. Still, we can appreciate his interest in multielectronic atoms. We cannot rule out the idea that Fermi's main motivation was more to solve the helium problem than that of ideal gases. Indeed, Dirac saw in Fermi's paper an application to electronic systems, not the foundations of a new statistics.

In the period considered, the attribution and range of applicability of each of the two statistics was widely discussed, generally relegating the Bose-Einstein to radiation and the Fermi-Dirac to matter. ${ }^{22}$ But of course nothing was settled in those first steps of quantum statistics. We have shown that the same can be said for the new status of elementary particles and their individuality: that does not seem to have been an issue of much concern for the physicists engaged in constructing quantum mechanics. The indistinguishability among molecules was not a determining and significant concept in that period (1925-1926), but a concept used in modern readings of those first developments; except for honorable exceptions, like that of Langevin. In a future work, we will return in greater detail to this question, addressing more specifically the works by Dirac and Heisenberg [9,29].

Acknowledgements This research was supported by the Spanish Ministerio de Ciencia, Innovación y Universidades Under Contract PID2019-105131GB-I00. We also thank to Anthony Duncan, Gonzalo Gimeno, Mercedes Xipell and Michael Bunn for his assistance during the preparation of this paper.

Funding Open Access funding provided thanks to the CRUE-CSIC agreement with Springer Nature.

Open Access This article is licensed under a Creative Commons Attribution 4.0 International License, which permits use, sharing, adaptation, distribution and reproduction in any medium or format, as long as you give appropriate credit to the original author(s) and the source, provide a link to the Creative Commons licence, and indicate if changes were made. The images or other third party material in this article are included in the article's Creative Commons licence, unless indicated otherwise in a credit line to the material. If material is not included in the article's Creative Commons licence and your intended use is not permitted by statutory regulation or exceeds the permitted use, you will need to obtain permission directly from the copyright holder. To view a copy of this licence, visit http://creativecommons.org/licenses/by/4.0/.

\section{References}

1. Belloni, L. 1999. On Fermi's route to Fermi-Dirac statistics. American Journal of Physics 15: 102-109.

2. Bergia, S. 1987. Who discovered the Bose-Einstein statistics?. In García Doncel, M. et al. (ed.) (1987), Symmetries in physics (1600-1980). Bellaterra (Barcelona): Seminari d'Història de les Ciències, UAB, 221-250.

3. Bernardini, C., Bonolis, L., Ghisu, D., Savelli, D. and Falera, L. 2003. Proceedings of the International Conference "Enrico Fermi and the Universe of Physics." Rome, September 29-October 2, 2001. Rome: ENEA.

4. Bernardini, C. and Bonolis, L. 2004. Enrico Fermi: his work and legacy. Bologna: Springer.

5. Brody, E. 1921. Zur theoretischen Bestimmung der chemischen Konstante einatomiger Gase. Zeitschrift für Physik 6: 79-83.

6. Como conference. 1928. Atti del Congresso Internazionale dei Fisici. 11-20 Settembre 192\%. Bologna: Nicola Zanichelli.

7. De Gregorio, A. and Sebastiani, F. 2010. Enrico Fermi and the old quantum physics. Quaderni di storia della fisica 16: 81-101. We cite the arxiv version in arXiv:0904.0260.

8. Dieks, D. 1990. Quantum statistics, identical particles and correlations. Synthese 82: 127-155.

9. Dirac, P. A. M. 1926. On the theory of quantum mechanics. Proceedings of the Royal Society A 112: 661-677.

10. Duncan, A. and Janssen, M. 2019. Constructing quantum mechanics. Oxford: Oxford University Press.

11. Eckert, M. 1987. Propaganda in science: Sommerfeld and the spread of the electron theory of metals. Historical Studies in the Physical Sciences 17: 191-233.

12. Ehrenfest, P. and Trkal, V. 1920. Deduction of the dissociation equilibrium from the theory of quanta and a calculation of the chemical constant based on this. Koninklijke Akademie van Wetenschappen te Amsterdam. Section of Sciences. Proceedings 23: 162-183. Reprinted in [31, pp. 414-435].

13. Ehrenfest, P. 1927. Relation between the reciprocal impenetrability of matter and Pauli exclusion principle. Text and correction. Nature 119: 196 and 602. Reprinted in [31, p. 546].

14. Ehrenfest, P. and Uhlenbeck, G. 1927. Die wellenmechanische Interpretation der Boltzmannschen Statistik neben der der neueren Statistiken. Zeitschrift für Physik 41: 24-26. Reprinted in [31, pp. 536-538].

15. Einstein, A. 1924. Quantentheorie des einatomigen idealen Gases. Sitzungsberichte der Preußischen Akademie der Wissenschaften (Berlin). Physikalisch-mathematische Klasse: 261-267. Reprinted in [32, pp. 433-441]. English translation in [33, pp. 371-383].

22 Nowadays, despite admitting that some mass particles can be governed by boson statistics, elementary massive particles are fermions, and those elementary particles that correspond to radiation, bosons. 
16. Einstein, A. 1925. Quantentheorie des einatomigen idealen Gases. Zweite Abhandlung. Sitzungsberichte der Preußischen Akademie der Wissenschaften (Berlin). Physikalisch-mathematische Klasse: 3-14. Reprinted in [32, pp. 580-594]. English translation in [33, pp. 276-283].

17. Fermi, E. 1923a. Il principio delle adiabatiche ed i sistemi che non ammettono coordinate angolari. Nuovo Cimento 25: 171-175. Reprinted in [25, pp. 88-91].

18. Fermi, E. 1923b. Beweis dass ein mechanisches Normalsystem im allgemeinen quasi-ergodisch ist. Physikalische Zeitschrift 24: 261-265. Reprinted in [25, pp. 79-86].

19. Fermi, E. 1923c. Alcuni teoremi di meccanica analitica importanti per la teoria dei quanti. Nuovo Cimento 25: $271-285$. Reprinted in [25, pp. 92-101].

20. Fermi, E. 1923d. Sopra la teoria si Stern della constante assoluta dell'entropia di un gas perfetto monoatomico. Rendiconti Lincei 32: 395-398. Reprinted in [25, pp. 114-117].

21. Fermi, E. 1924. Considerazioni sulla quantizzazione dei sistema che contengono degli elementi identici. Nuovo Cimento I: $145-152$. Reprinted in [25, pp. 124-129].

22. Fermi, E. 1926a. Sulla quantizzazione del gas perfetto monoatomico. Rendiconti Lincei 3: 145-149. Reprinted in [25, pp. 181-184]

23. Fermi, E. 1926b. Zur Quantelung des idealen einatomigen Gases. Zeitschrift für Physik 36: 902-912. Reprinted in [25, pp. 186-195].

24. Fermi, E. 1927. Un metodo statistico per la determinazione di alcune propietà dell'atomo. Rend. Lincei 6: 602-607. Reprinted in [25, pp. 278-282].

25. Fermi, E. 1965. Note e memorie: Collected papers. Roma: Academia Nazionale dei Lincei, University of Chicago Press. Vol 1.

26. Ford, G. W. 2009. George Eugene Uhlenbeck, 1900-1988. A biographical memoir. Washington: National Academy of Sciences.

27. Fowler, R. H. 1926a. General forms of statistical mechanics with special reference to the requirements of the new quantum mechanics. Proceedings of the Royal Soceity A 113: 432-449.

28. Fowler, R. H. 1926b. On dense matter. Monthly Notices of the Royal Astronomical Society 87: $114-122$.

29. Heisenberg, W. 1926. Mehrkörperproblem und Resonanz in der Quantenmechanik. Zeitschrift für Physik 38: 411-426.

30. Hoddeson, L., Baym, G. and Eckert, M. 1992. The Development of the Quantum Mechanical Electron Theory of Metals, 1926-1933. In Hoddeson, L., Braun, E., Teichmann, J. and Weart, S., Out of the Crystal Maze. Chaptes of the History of Solid-State Physics." New York: Oxford University Press, 88-181.

31. Klein, M. J. (ed.) 1959. Paul Ehrenfest. Collected scientific papers (with an introduction by H. B. G. Casimir). Amsterdam: North-Holland.

32. Kormos Buchwald, D., Illy, J. and Rosenkranz, Z. Sauer, T. and Moses, O. 2015a. The collected papers of Albert Einstein. Volume 14: The Berlin years: Writings and correspondence, April 1923-May 1925. Princeton: Princeton University Press.

33. Kormos Buchwald, D., Illy, J., Rosenkranz, Z., Sauer, T. and Moses, O. 2015b. The collected papers of Albert Einstein. Volume 14: The Berlin years: Writings and correspondence, April 1923-May 1925 (English Translation Supplement). Princeton: Princeton University Press.

34. Kragh, H. S. 1990. Dirac. A scientific biography. Cambridge: Cambridge University Press.

35. Kramers, H. A. 1937. Didaktisches zur Verwendung der grand Ensembles in der Statistik. Koninklijke Akademie van Wetenschappen te Amsterdam. Section of Sciences. Proceedings 41: 10-24.

36. Monaldi, D. 2009. A note on the prehistory of indistinguishabilty particles. Studies in History and Philosophy of Modern Physics 40: 383-394.

37. Pais, A. 1982. 'Subtle is the Lord...' The science and the life of Albert Einstein. Oxford: Oxford University Press.

38. Pauli, W. 1925. Über den Zusammenhang des Abschlusses der Elektronengruppen im Atom mit der Komplexstruktur der Spektren. Zeitschrift für Physik 31: 765-783.

39. Pauli, W. 1927. Über Gasentartung und Paramagnetismus. Zeitschrift für Physik 41: 81-102.

40. Pérez, E. and Sauer, T. 2010. Einstein's quantum theory of monatomic ideal gas: non-statistical arguments for a new statistics. Archive for History of Exact Sciences 64: 561-612.

41. Planck, M. 1916. Über die Absolute Entropie einatomiger Körper. Sitzungsberichte der Preußischen Akademie der Wissenschaften (Berlin). Physikalisch-mathematische Klasse: 653-667.

42. Planck, M. 1925. Zur Frage der Quantelung einatomiger Gase. Sitzungsberichte der Preußischen Akademie der Wissenschaften (Berlin). Physikalisch-mathematische Klasse: 49-57.

43. Sackur, O. 1913. Die universelle Bedeutung des sogenannten elementaren Wirkungsquanums. Annalen der Physik 40: 67-86.

44. Saunders, S. 2020. The concept ìndistinguishable. Studies in History and Philosophy of Science. Part B: Studies in the History and Philosophy of Modern Physics 71: 37-59.

45. Sebastiani, F. and Cordella, F. 2000. Sul percorso di Fermi verso la statistica quantica. Il nuovo saggiatore 16: 11-23.

46. Sebastiani, F. and Cordella, F. 2003. Fermi towards quantum statistics (1923-1925). In [Bernardini et al. 2001], 71-96.

47. Scherrer, P. 1916. Das ideale Gas als bedingt periodisches System im Sinne der Quantentheorie. Göttinger Nachrichten: 154-159.

48. Seglar, P. and Pérez, E. 2015. A grasp of identity. European Journal of Physics 36035016.

49. Seglar, P. and Pérez, E. 2018. Física estadística. De estados y partículas: una mirada nueva a viejas controversias. Barcelona: Edicions UB. 
50. Segrè, E. 1970. Enrico Fermi, physicist. Chicago: University of Chicago Press.

51. Solvay. 1927. Electrons et photons. Rapports et discussions du cinquiéme conseil de physique tenu a Bruxelles du 24 au 29 octobre 192\%. Paris: Gautier-Villars.

52. Sommerfeld, A. 1927. Zur Elektronentheorie der Metalle. Die Naturwissenschaften 15: 825-832.

53. Sommerfeld, A. 1928a. Zur Elektronentheorie der Metalle. Die Naturwissenschaften 21: 374-381.

54. Sommerfeld, A. 1928b. Zur Elektronentheorie der Metalle auf Grund der Fermischen Statistik. Zeitschrift für Physik 47: 1-32 and 43-60.

55. Sommerfeld, A. 1928c. Zur Elektronentheorie der Metalle und des Volta-Effektes nach der Fermi'schen Statistik. In [Como 1928], 449-473.

56. Stern, O. 1913. Zur kinetischen Theorie des Dampfdrucks einatomiger fester Stoffer und über die Entropiekonstante einatomiger Gase. Physikalische Zeitschrift 14: 629-632.

57. Stern, O. 1919. Zusammenfassender Bericht über die Molekulartheorie des Dampfdruckes fester Stoffe und ihre Bedeutung für die Berechnung chemischer Konstanten. Zeitschrift für Elektrochemie 25: 66-80.

58. Stoner, E. C. 1924. The distribution of electrons among electronic levels. Philosophical Magazine 48: 719-736.

59. Tetrode, H. 1912. Die chemische Konstante der Gase und das elementare Wirkungsquantum. Annalen der Physik 38: 434-442, 39: 255-256.

60. Thomas, L. H. 1926. The calculation of atomic fields. Proceedings of the Cambridge Philosophical Society 23: 542-548.

61. Wigner, E. 1927. Über nicht-kombinierende Terme in der neueren Quantentheorie. Zeitschrift für Physik 40: 492-500, 883-892. 